\title{
Optimizing the Diagnostic Strategy to Identify Genetic Abnormalities in Miscarriage
}

\author{
Jong-Mi Lee ${ }^{1,2}$. So Young Shin ${ }^{3}$ Guk Won Kim ${ }^{4} \cdot$ Woo Jeng Kim ${ }^{4}$ Jeong Ha Wie ${ }^{4}$. Subeen Hong ${ }^{4}$. Dain Kang ${ }^{2}$. \\ Hayoung Choi ${ }^{2} \cdot$ Jisook Yim ${ }^{1,2} \cdot$ Yonggoo Kim ${ }^{1,2} \cdot$ Myungshin Kim ${ }^{1,2}$ (D) In Yang Park ${ }^{4}$
}

Accepted: 6 March 2021 / Published online: 1 April 2021

(c) The Author(s) 2021

\begin{abstract}
Introduction The single most common cause of miscarriage is genetic abnormality.

Objective We conducted a prospective cohort study to compare the performance of conventional karyotyping and chromosomal microarray analysis (CMA) using array comparative genomic hybridization (array-CGH) and single nucleotide polymorphism array (SNP-array) to identify genetic abnormalities in miscarriage specimens.

Methods A total of 63 miscarriage specimens were included. Conventional karyotyping, array-CGH, and SNP-array were performed and the results compared.

Results Genetic abnormalities were detected in 31 cases $(49.2 \%)$ by at least one testing modality. Single autosomal trisomy was the most common defect (71.0\%), followed by polyploidy (16.1\%), multiple aneuploidy (9.7\%), and monosomy X (3.2\%). Mosaicisms were identified in four cases and confirmed by fluorescence in situ hybridization (FISH) using appropriate probes. SNP-array had a higher detection rate of genetic abnormalities than array-CGH (93.5 vs. 77.4\%), and conventional karyotyping had the lowest detection rate (76.0\%). SNP-array enabled the detection of all types of genetic abnormalities, including polyploidy.

Conclusions Although conventional karyotyping and FISH are still needed, SNP-array represents the first choice for miscarriage because the technique showed excellent performance in the detection of genetic abnormalities and minimized the probability of testing failure as well as time, costs, and labor.
\end{abstract}

Myungshin Kim and In Yang Park contributed equally to this work.

\footnotetext{
Myungshin Kim

microkim@catholic.ac.kr

In Yang Park

ooooobbbbb@catholic.ac.kr

1 Department of Laboratory Medicine, College of Medicine, The Catholic University of Korea, 222 Banpodaero, Seocho-gu, Seoul 06591, South Korea

2 Catholic Genetic Laboratory Center, Seoul St. Mary's Hospital, College of Medicine, The Catholic University of Korea, 222 Banpodaero, Seocho-gu, Seoul 06591, South Korea

3 Department of Obstetrics and Gynecology, College of Medicine, Kyung Hee University Hospital, Kyung Hee University, 23, Kyung Hee Dae-ro, Dongdaemun-gu, Seoul 02447, South Korea

4 Department of Obstetrics and Gynecology, College of Medicine, The Catholic University of Korea, 222 Banpodaero, Seocho-gu, Seoul 06591, South Korea

\begin{tabular}{l}
\hline $\begin{array}{l}\text { Myungshin Kim } \\
\text { microkim@ @ catholic.ac.kr }\end{array}$ \\
In Yang Park \\
ooooobbbbb@ catholic.ac.kr \\
$1 \quad \begin{array}{l}\text { Department of Laboratory Medicine, College of Medicine, } \\
\text { The Catholic University of Korea, 222 Banpodaero, } \\
\text { Seocho-gu, Seoul 06591, South Korea }\end{array}$ \\
$2 \begin{array}{l}\text { Catholic Genetic Laboratory Center, Seoul St. Mary's } \\
\text { Hospital, College of Medicine, The Catholic University } \\
\text { of Korea, 222 Banpodaero, Seocho-gu, Seoul 06591, }\end{array}$ \\
South Korea \\
Department of Obstetrics and Gynecology, College \\
of Medicine, Kyung Hee University Hospital, Kyung Hee \\
University, 23, Kyung Hee Dae-ro, Dongdaemun-gu, \\
Seoul 02447, South Korea \\
Department of Obstetrics and Gynecology, College \\
of Medicine, The Catholic University of Korea, 222 \\
Banpodaero, Seocho-gu, Seoul 06591, South Korea
\end{tabular}
}

\section{Key Points}

This article provides a diagnostic strategy to identify genetic abnormalities in miscarriage by comparing results from conventional karyotyping, array comparative genomic hybridization (array-CGH), and single nucleotide polymorphism array (SNP-array).

SNP-array had the best detection capacity, followed by array-CGH and conventional karyotyping.

SNP-array represents the first choice because it showed excellent performance in the detection of genetic abnormalities, including polyploidy. 


\section{Introduction}

Miscarriage is defined as the loss of pregnancy during the first 20 weeks' gestation. It occurs in about $15-25 \%$ of all clinically recognized pregnancies [1]. The single most common cause of miscarriage is chromosomal abnormality, which accounts for about $50 \%$ of cases [1-5], particularly autosomal trisomy, which constitutes the majority of cases, followed by triploidy, monosomy $\mathrm{X}$, tetraploidy, and structural anomalies [3, 6-8]. Genetic testing of miscarriage specimens provides valuable clinical information and allows couples to seek appropriate management, genetic counseling, and emotional support [2,9]. Conventional karyotyping has been the most commonly used genetic test for the determination of pregnancy loss for over 30 years and is considered the gold standard. However, this technique has several significant limitations, including high rates of culture failure and bacterial and maternal cell contamination (MCC) and is a laborintensive and time-consuming procedure. It is also unable to detect abnormalities $<3 \mathrm{Mb}$ [3, 9-12].

Currently, chromosomal microarray (CMA) is an alternative to conventional karyotyping. It has become the first-line test for the diagnosis of autism, developmental delay, intellectual disability, and congenital anomalies [13-15]. Two types of CMA are available as genetic tests in a clinical laboratory: array comparative genomic hybridization (array-CGH) and single nucleotide polymorphism array (SNP-array). Both molecular techniques offer relatively fast and objective results and require minimal tissue. They also facilitate the detection of submicroscopic deletions and duplications, known as copy number variations $(\mathrm{CNVs})$, which are missed by conventional karyotyping. SNP-array provides additional information of uniparental disomy and consanguinity though analysis of SNPs [6, 11, 16, 17]. Nevertheless, CMAs are associated with several limitations, including the inability to detect balanced chromosomal rearrangements and low-level mosaicism, and the array-CGH does not reliably detect all polyploidies [17, 18]. The demand for CMA in genetic testing of miscarriage specimens is increasing, but few studies have performed headto-head comparisons of conventional and new techniques [9, $10,19]$.

We designed a prospective study to investigate the incidence and spectrum of genetic abnormalities in miscarriage specimens in a real-life clinic at a single Korean institution and to provide comparative data from conventional karyotyping, array-CGH, and SNP-array to develop an optimal strategy.

\section{Study Design}

\subsection{Clinical Samples}

This prospective study was performed at Seoul St. Mary's Hospital, College of Medicine, The Catholic University of Korea (Seoul, South Korea) between November 2017 and July 2018. A total of 63 fetuses that underwent spontaneous fetal demise before 18 weeks of gestation were included. The miscarriage was diagnosed by transvaginal ultrasound and confirmed by repeat ultrasound at periodic time intervals. The diagnosis was conducted according to international guidelines [20]. The exclusion criteria were multiple pregnancies, suspected molar pregnancy, exceeding 20 gestational weeks by the last menstrual period, exceeding 18 gestational weeks by sonographic dating, and insufficient specimen. The evacuated product of conception was carefully dissected to identify chorionic villi (CV) to minimize MCC. CV were equally divided into two samples and used for cell culture via conventional karyotyping and DNA extraction for array-CGH and SNP-array. The study was conducted in accordance with the ethical guidelines of the Declaration of Helsinki and was approved by the institutional review board/ethics committee of The Catholic University of Korea (KC18SESI0075). Written informed consent was obtained from all participants.

\subsection{Conventional Karyotyping}

Conventional karyotyping was performed on G-banded metaphase chromosomes of cultured $\mathrm{CV}$ using routine techniques. Briefly, the released CV was cleaned, chopped, and briefly treated with trypsin-EDTA (Gibco, UK). Prewarmed Amniomax II medium (Gibco, UK) was added for further incubation, and microscopic inspection was performed daily to determine the harvest time. Bacterial contamination was diagnosed when culture media became turbid during incubation and microscopic examination showed moving microorganisms. After prolonged incubation, cells were harvested using colcemid (Fusifilm, Japan) and trypsin-EDTA. Metaphase chromosomes were treated with a methanol/glacial acetic acid fixative (Duksan Pure Chemicals, Korea). G-banding was done using Giemsa solution (Merk, Germany). Karyotypes were described and interpreted according to the International System for Human Cytogenomic Nomenclature 2016 [21].

\subsection{Array-CGH}

Array-CGH analysis was performed using a SurePrint G3 Human CGH Microarray $8 \times 60 \mathrm{~K}$ kit (Agilent Technologies, 
Santa Clara, CA, USA) as described previously [15, 22]. It contains 62,976 oligonucleotide probes spaced at $41 \mathrm{Kbp}$ intervals (median probe spacing) throughout the genome. Control DNA (Promega Corp., Nepean, Canada) was used as the reference DNA. DNA digestion, labeling, and hybridization were performed following the manufacturer's instructions. Scanned images were quantified using Agilent Feature Extraction software (v10.0), and the resulting data were imported into Agilent Genomic Workbench 7.0.4.0 software for visualization. CNVs were detected using the Aberration Detection Method-2 (ADM-2) algorithm. All genomic coordinates were based on human genome build hg19/GRCh37.

\subsection{SNP-Array}

SNP-array analysis was performed using HumanCytoSNP-12 v2.1 BeadChip Kit (Illumina, San Diego, CA, USA) according to the manufacturer's instructions. This kit consists of genome-wide SNP markers targeting all regions of known cytogenetic importance. Images were captured on NextSeq550, and data were primarily analyzed using Illumina's GenomeStudio. Resolution was set at $0.1 \mathrm{Mb}$ for deletion, $0.5 \mathrm{Mb}$ for gain, and $3 \mathrm{Mb}$ for loss of heterozygosity/ absence of heterozygosity. The proportion of SNP alleles (B) in the total copy number was presented as B allele frequency (BAF). BAF values of 0 and 1 indicate homozygous alleles ( $\mathrm{AA}$ and $\mathrm{BB}$, respectively), whereas 0.5 indicates a heterozygous allele $(A B)$. In case of a single copy gain of chromosome, two extra $\mathrm{BAF}$ bands appear at the value of $0.33(\mathrm{AAB})$ and $0.67(\mathrm{ABB})$ along with 0 (AAA) and 1 (BBB) [23]. The number of lines in the BAF chart can be used to recognize both polyploidy and the copy numbers. Secondary analysis was carried out using online resources such as DECIPHER (http://decipher.sanger.ac.uk/), OMIM (http://www.ncbi.nlm.nih.gov/omim), and UPD with cross reference to UCSC (http://genome.ucsc.edu/) and the database of Genomic Variants (http://projects.tcag.ca/variation/).

Interpretation and reporting followed American College of Medical Genetics Standards and Guidelines for constitutional cytogenomic microarray analysis, including postnatal and prenatal applications [24]. Both CMAs were repeated using new DNA isolated from the stored specimen in cases of discrepancy between the CMAs and conventional karyotyping to exclude random sampling error.

\subsection{Fluorescence In Situ Hybridization}

Fluorescence in situ hybridization (FISH) of cultured cells was performed where there was discordance between conventional karyotyping and CMAs. It was carried out using a standard method as previously described [22] with commercially available probes: BCR/ABL(ABL1) Translocation, Dual Fusion (9q34.11-q34.12; 22q11.22-q11.23, Cytocell, UK), Dual-Labeled Satellite Probe Sets (Xp11.1q11.1; Yq12, Cytocell), and XL 20q12/20qter plus (20q12; 20q13.3, MetaSystems, Germany).

\subsection{Statistical Analysis}

The Kruskal-Wallis test was used to compare the differences in maternal age, gestational age, and history of fetal loss. The chi-squared test was used to compare the detection rates with the three methods. Fisher's exact test was used to compare the two methods (Fig. 1). A significance level of 0.05 was used for the analysis. Analyses were performed using MedCalc (v.19.5.1).

\section{Results}

\subsection{Cohort Demographics According to the Presence of Genetic Abnormality}

The mean maternal age at the time of miscarriage diagnosis was 36.6 years, and the mean gestational age at the time of uterine evacuation was about 9 weeks $(5+3$ to $17+2$ weeks). A prior pregnancy loss had been experienced by $39.7 \%$ (25/63) of participants. Conventional karyotyping failed in $22.2 \%$ (14/63) mainly because of bacterial contamination (78.6\% [11/14]). Array-CGH and SNP-array were successfully performed in all cases. Genetic abnormalities were identified in $49.2 \%$ (31/63) of cases using at least one

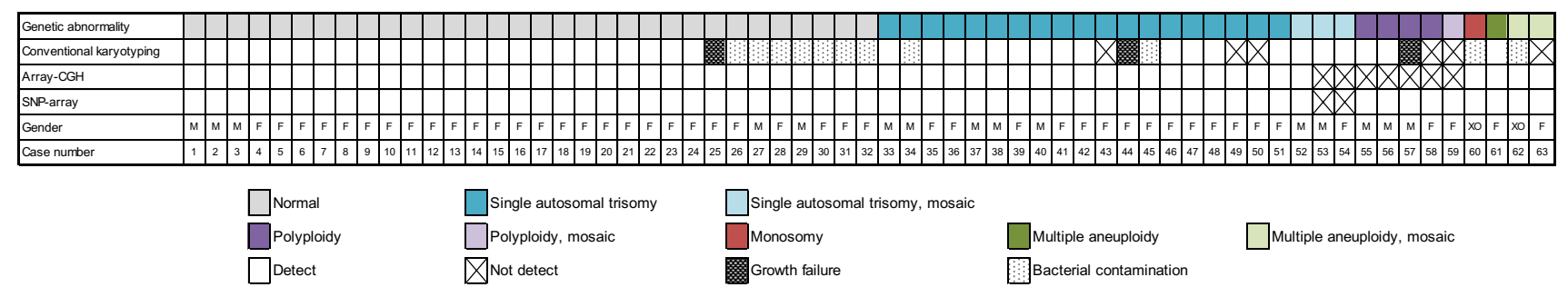

Fig. 1 Genetic results of 63 miscarriages identified using three different methods. Each column represents the results of the case with the corresponding number. $C G H$ comparative genomic hybridization, $F$ female, $M$ male, $S N P$ single nucleotide polymorphism 
of the three techniques, including conventional karyotyping, array-CGH, and SNP-array. One case showing a balanced pericentric inversion of chromosome 9 [inv(9)(p12q13)] was considered normal. We compared the demographic differences between the two groups and found no significant differences in maternal age and number of prior miscarriages. However, gestational age was significantly higher in cases of genetic abnormality than in those without such abnormality $(9+4$ vs. $8+3$ weeks, $P=0.01)$ (Table 1$)$.

\subsection{Characteristics of Identified Genetic Abnormalities}

Among the 31 cases of genetic abnormality, 19 (61.3\%) showed a female genotype. Single autosomal trisomy was the most common (71.0\% [22/31]), followed by polyploidy $(16.1 \%[5 / 31])$, multiple aneuploidy $(9.7 \%$ [3/31]), and monosomy X (3.2\% [1/31]). Mosaicism was defined when any of the methods identified the presence of two or more chromosomally distinct cell lines. A total of six cases with mosaicism were identified, including three single autosomal trisomies (case 52, 53, 54), two multiple aneuploidies (case 62 and 63), and one polyploidy (case 59). Trisomy $22(27.3 \%$ [6/22]) was the most frequently observed single autosomal trisomy, followed by trisomy $16(22.7 \%$ [5/22]), trisomy 15 (13.6\% [3/22]), and trisomy $18(9.1 \%$ [2/22]). Other trisomies involved chromosomes 2, 3, 7, 17, 20, and $21(4.5 \%$ each [1/22]) (Fig. 2, Table 1 in the electronic supplementary material [ESM]). Three cases of multiple aneuploidy included trisomies 7, 18, and 20; monosomy X and trisomy 14; and trisomies 15 and 21. Among 32 cases without detectable genetic abnormality, we found gender distribution to be skewed: $84.4 \%$ (27/32) were female and males accounted for only $15.6 \%(5 / 32)$.

Table 1 Cohort characteristics

\begin{tabular}{llll}
\hline Characteristics & Total $(N=63)$ & Normal $(N=32)$ & Abnormal $(N=31)$ \\
\hline Maternal age, years & $36.6 \pm 4.0$ & $36.1 \pm 3.9$ & $37.0 \pm 4.2$ \\
Gestational age, days & $62.6 \pm 15.4$ & $58.6 \pm 15.1$ & $66.7 \pm 14.8$ \\
Prior miscarriages & $0.5 \pm 0.8$ & $0.4 \pm 0.7$ & $0.6 \pm 0.8$ \\
0 & $38(60.3)$ & $22(68.8)$ & $16(51.6)$ \\
1 & $21(33.3)$ & $8(25.0)$ & $13(41.9)$ \\
2 & $2(3.2)$ & $1(3.1)$ & $1(3.2)$ \\
3 & $1(1.6)$ & $1(3.1)$ & 0.46 \\
4 & $1(1.6)$ & $0(0)$ & $1(3.2)$ \\
\hline
\end{tabular}

Data are presented as mean \pm standard deviation or $N(\%)$ unless otherwise indicated

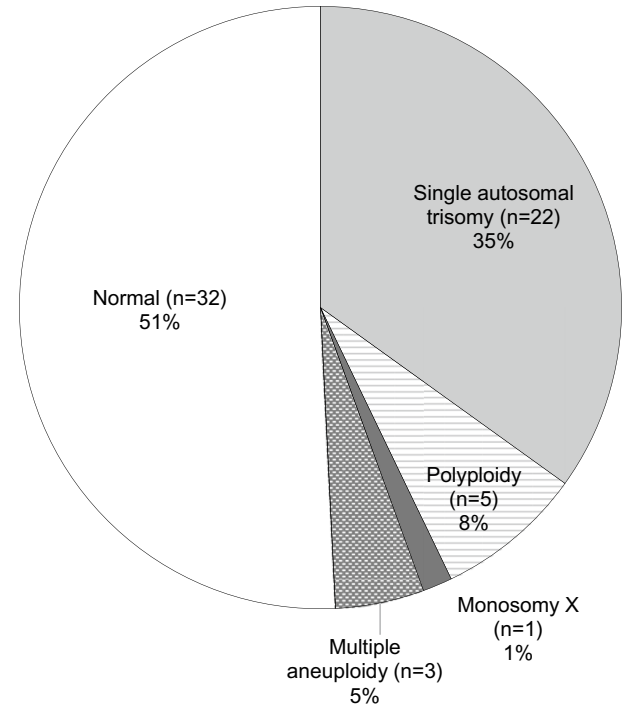

Fig. 2 Distribution of genetic abnormalities and aneuploidy
Frequency of aneuploidy

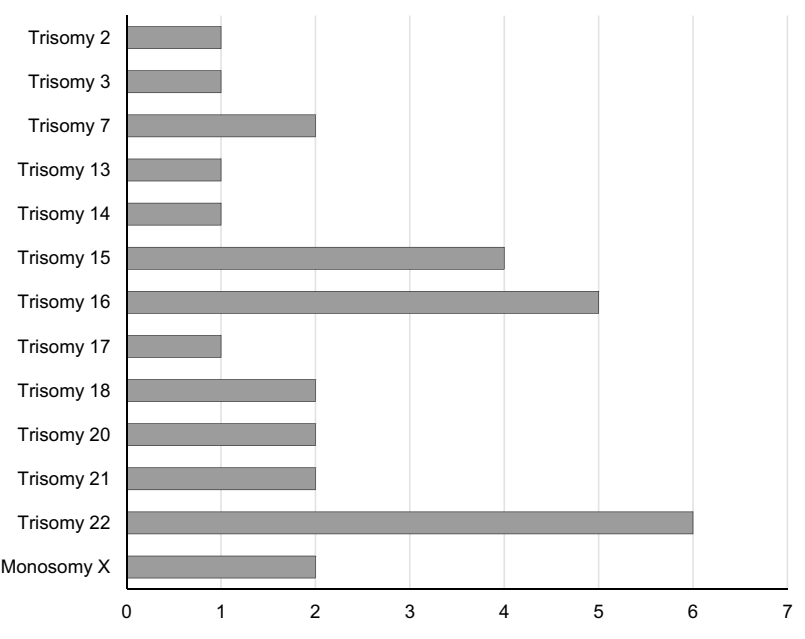




\subsection{Comparison of Conventional Karyotyping, Array-CGH, and SNP-Array}

We analyzed the capacity to detect genetic abnormalities in 31 cases. Sensitivity was significantly higher with SNParray $(93.5 \%$ [29/31]) than with array-CGH (77.4\% [24/31]) $(P=0.045)$. The analytical sensitivity of conventional karyotyping was the lowest $(76.0 \%$ [19/25]), even after cases of culture failure $(n=6)$ were excluded (Table 2 in the ESM). Random sampling errors occurred in three cases (case 35, 41, and 51); these were reanalyzed and corrected using newly isolated DNA samples and both CMAs. The CMAs initially showed normal female results, whereas conventional karyotyping showed single autosomal trisomy. Reanalysis using new samples showed results consistent with those of the conventional karyotyping. Based on the type of genetic abnormality, all 19 single autosomal trisomies were identified using both array-CGH and SNP-array, whereas conventional karyotyping failed to detect three of them $(15.8 \%$ [3/19]), as shown in Table 2 (cases 43, 49, and 50). We verified the presence of CMA-detected trisomies via FISH in three cases with available residual pellets after conventional karyotyping. Case 49 showed XY and trisomy 22 based on CMAs, whereas the results of conventional karyotyping were not consistent with those of normal females. FISH using probes specific for $\mathrm{X}$ and $\mathrm{Y}$ chromosomes were used to identify the $\mathrm{Y}$ chromosome, and $9 \%$ of cells were positive for $\mathrm{Y}$ chromosomes (red signals) (Fig. 3a). Thus, we revealed that the sex of the $\mathrm{CV}$ was male and the conventional karyotyping result was derived from maternal cells. Case 50 showed trisomy 22 based on CMA, but this was not detected with conventional karyotyping. Three copies

Table 2 Discordant results based from the three different methods

\begin{tabular}{|c|c|c|c|c|c|}
\hline Case & Genetic result & Conventional karyotyping & Array-CGH & SNP-array & FISH analysis \\
\hline 43 & Single autosomal trisomy & $46, X^{a}$ & $\operatorname{arr}(18) \times 3$ & $\operatorname{arr}(18) \times 3$ & \\
\hline 49 & Single autosomal trisomy & $46, X^{a}$ & $\operatorname{arr}(22) \times 3,(X, Y) \times 1$ & $\operatorname{arr}(22) \times 3,(X, Y) \times 1$ & $\begin{array}{c}\text { nuc ish }(\mathrm{DXZ1}, \mathrm{DYZ1}) \times 1[9 / 100] / \\
(\mathrm{DXZ} 1 \times 2, \mathrm{DYZ} 1 \times 0)[91 / 100]\end{array}$ \\
\hline 50 & Single autosomal trisomy & $46, \mathrm{XX}^{\mathrm{a}}$ & $\operatorname{arr}(22) \times 3$ & $\operatorname{arr}(22) \times 3$ & nuc ish $(\mathrm{BCR} \times 2, \mathrm{ABL} 1 \times 3)[10 / 100]$ \\
\hline 53 & $\begin{array}{l}\text { Single autosomal trisomy, } \\
\text { mosaic }\end{array}$ & $\begin{array}{l}\operatorname{mos} \\
\quad 47, X Y,+16[3] / 46, X Y[17]\end{array}$ & $\operatorname{arr}(1-22, X) \times 2^{a}$ & $\operatorname{arr}(1-22, X) \times 2^{a}$ & \\
\hline 54 & $\begin{array}{l}\text { Single autosomal trisomy, } \\
\text { mosaic }\end{array}$ & $\begin{array}{l}\operatorname{mos} \\
47, X X,+22[3] / 46, X X[17]\end{array}$ & $\operatorname{arr}(1-22, X) \times 2^{a}$ & $\operatorname{arr}(1-22, X) \times 2^{a}$ & \\
\hline 55 & Polyploidy & $69, \mathrm{XXY}$ & $\operatorname{arr}(1-22) \times 2,(\mathrm{X}, \mathrm{Y}) \times 1^{\mathrm{a}}$ & $\operatorname{arr}(1-22) \times 3,(\mathrm{X}) \times 2,(\mathrm{Y}) \times 1$ & \\
\hline 56 & Polyploidy & 71,XXYYY & $\operatorname{arr}(1-22) \times 2,(\mathrm{X}, \mathrm{Y}) \times 1^{\mathrm{a}}$ & $\operatorname{arr}(1-22) \times 3,(\mathrm{X}) \times 2,(\mathrm{Y}) \times 3$ & \\
\hline 57 & Polyploidy & Growth failure & $\operatorname{arr}(18) \times 3^{\mathrm{a}}$ & $\begin{array}{l}\operatorname{arr}(1-17,19- \\
\quad 22) \times 4,(18) \times 5,(\mathrm{X}) \times 3,(\mathrm{Y}) \times 1\end{array}$ & \\
\hline 58 & Polyploidy & $46, X^{a}$ & $\operatorname{arr}(1-22, X) \times 2^{a}$ & $\operatorname{arr}(1-22, X) \times 4$ & \\
\hline 59 & Polyploidy, mosaic & $46, \mathrm{XX}^{\mathrm{a}}$ & $\operatorname{arr}(1-22, X) \times 2^{a}$ & $\operatorname{arr}(1-22, X) \times 3$ & \\
\hline 63 & Multiple aneuploidy, mosaic & $47, \mathrm{XX},+18^{\mathrm{a}}$ & $\operatorname{arr}(7) \times 3,(18) \times 3,(20) \times 3$ & $\operatorname{arr}(7) \times 3,(18) \times 3,(20) \times 3$ & $\begin{array}{c}\text { nuc ish(D20S108,MYBL2) } \times 3[12 / 100] / \\
(\text { D20S108,MYBL2 }) \times 4[15 / 100]\end{array}$ \\
\hline
\end{tabular}

$C G H$ comparative genomic hybridization, FISH fluorescence in situ hybridization, SNP single nucleotide polymorphism ${ }^{\text {a }}$ Results requiring correction
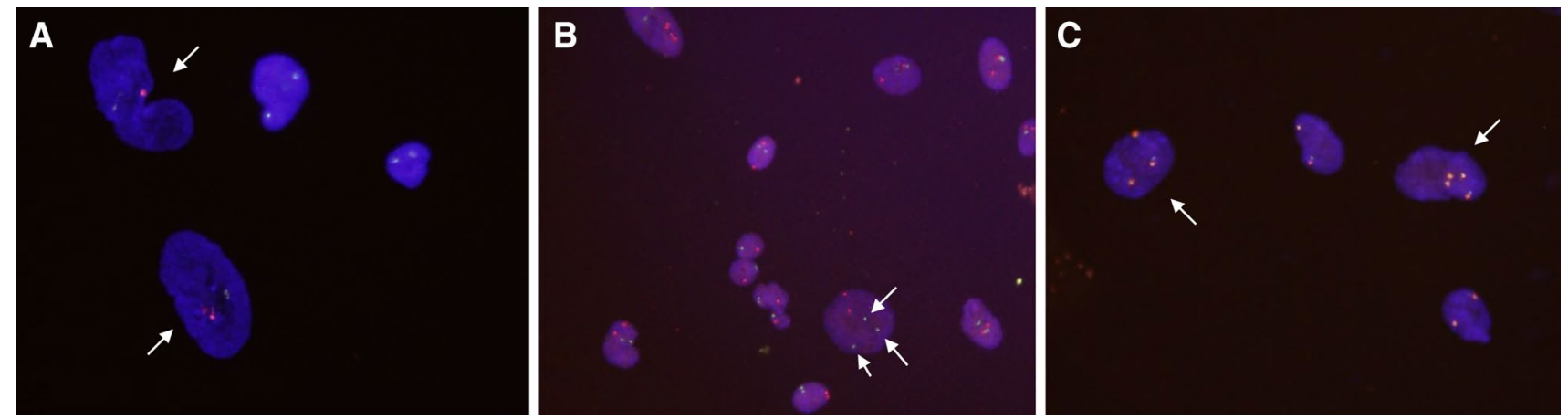

Fig. 3 Fluorescence in situ hybridization (FISH) using residual pellet after conventional karyotyping. (A) XY FISH showing 9\% of cells with Y chromosomes (red). (B) BCR-ABL1 FISH indicating $10 \%$ of the cells with extra copies of chromosome 22 (green, arrows). (C) 20q FISH showing three (left) and four (right) signals 
of chromosome 22 (green signals) were observed in $10 \%$ of cells using FISH analysis (Fig. 3b), suggesting possible sampling error (accidental overcrowding of specific cell population), tissue mosaicism (more than one type of cell population), or culture artifact (domination of viable cell population). In cases 53 and 54, low-level mosaic trisomy (15\% [3/20] metaphases) was detected via conventional karyotyping but not via CMA because of technical limitations. SNP-array enabled the detection of all cases of polyploidy (cases 55-59), including one mosaic polyploidy. However, none of these was detected by array-CGH. Conventional karyotyping was available in four cases, two of which corresponded to the results found with SNP-array; however, the other two revealed 46,XX, suggesting culture artifact because of more viable maternal cells. One interesting case of multiple aneuploidy (case 63 with trisomies 7, 18, and 20) was identified by both CMAs, whereas conventional karyotyping recognized only trisomy 18 . Additional FISH revealed three and four copies of chromosome 20 in 12 and $15 \%$ of cells, respectively (Fig. 3c), supporting the CMA findings (Fig. 4).

\section{Discussion}

In the present study, we diagnosed a series of genetic abnormalities in spontaneous miscarriage using three different techniques, including two types of CMAs and conventional karyotyping. This is one of the largest studies to date comparing the three different modalities using miscarriage samples. Genetic abnormalities were observed in $49.2 \%$ of miscarriages, which was in line with results from previous studies $[19,25,26]$, as was the overall distribution of genetic abnormalities in our cohort. Single autosomal trisomy was predominant, followed by polyploidy, multiple aneuploidy, and monosomy X. Among the trisomies, trisomies 22, 16, and 15 were prevalent. Cases with genetic abnormalities showed advanced gestational age compared with those without genetic abnormalities. Studies have revealed that genetic abnormalities are more likely to be found in early miscarriages [27, 28]. We reviewed 12 cases in fetal stages (more than $10+0$ weeks) and found that nine (75\%) had genetic abnormalities, including trisomy 18 , trisomy 21 , and monosomy X, which were commonly associated with relatively longer survival $[8,29]$. Conventional karyotyping is a quintessential aspect of evaluations of miscarriage because chromosomal abnormalities are a major contributing factor in early miscarriages $[4,30]$. Our laboratory, which belongs to a tertiary hospital and has rich experience in cytogenetic studies, recorded a culture success rate of $77.8 \%$, which is within the general success rate of $60-90 \%[7,11]$. The low success rate was due to culture failure, mainly bacterial contamination and poor viability of fetal cells in the $\mathrm{CV}$ $[8,9,12]$. Previous studies have shown that $15 \%$ of early miscarriages and $66 \%$ of late miscarriages are attributed to infections [31, 32]. The majority of culture failure in this study was associated with bacterial contamination, which supported the linkage between infection and miscarriage [33]. On the other hand, CMAs were conducted in all cases, and both CMAs satisfactorily detected aneuploidy. However, ploidy changes were shown as normal with the array-CGH profile, since array-CGH detects copy number imbalance by comparing the fluorescent intensity of test DNA with that of the control after labeling adjusted the same amount of DNA [34, 35]. Therefore,

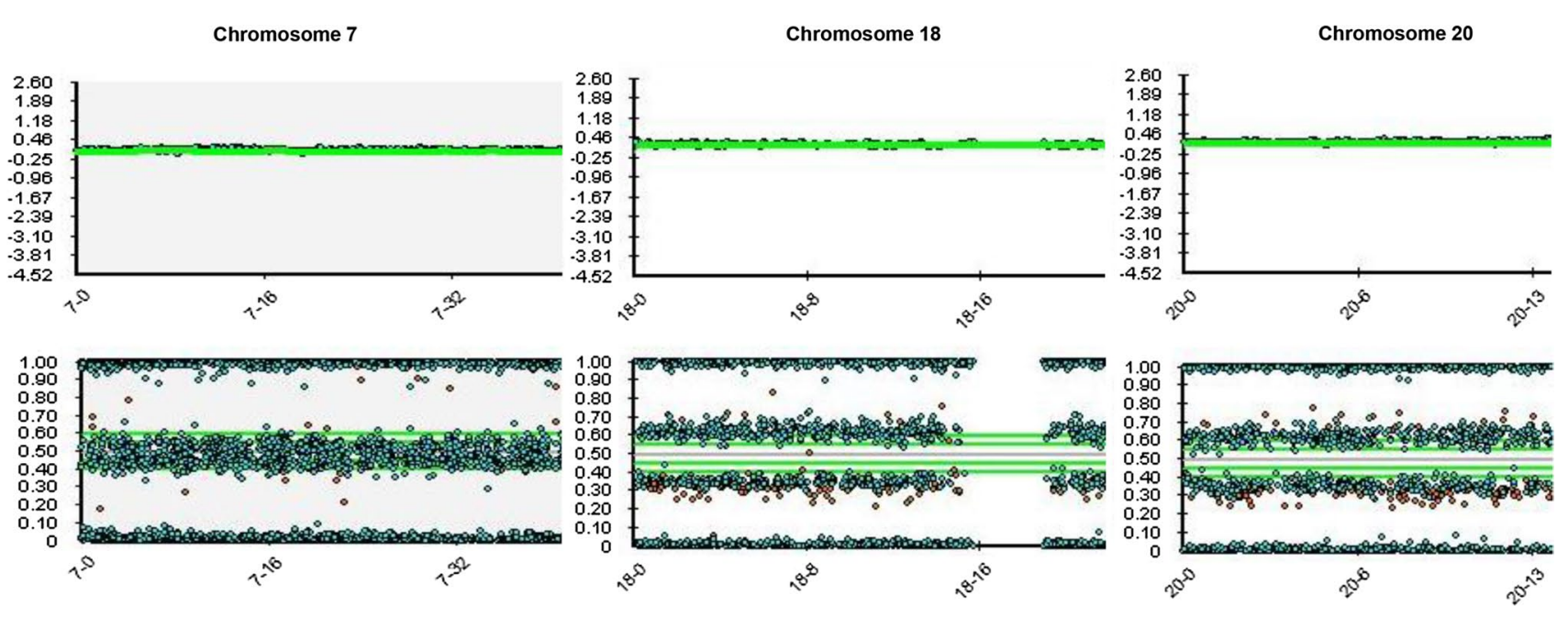

Fig. 4 Log R ratio (upper) and B allele (lower) plots derived from single nucleotide polymorphism (SNP)-array indicate mosaic trisomy 7, trisomy 18 , and trisomy 20 
further investigation using other techniques such as microsatellite analysis, FISH, and conventional karyotyping is required. Conversely, the SNP-array recognizes polyploidy by evaluating the BAF plot.

The SNP-array showed a 16\% higher detection potential than array-CGH. These findings are in line with a study suggesting that SNP-array yielded the best detection rates (93\%), and the other two methods were similar (85\%) [9]. We carefully evaluated cases showing discordant results using additional FISH experiments. FISH revealed that some conventional karyotyping could not effectively analyze fetal cells because of the selective overgrowth of viable maternal cells. Studies have shown that MCC constituted about $29.0-89.7 \%$ of normal female karyotype [12, 36, 37]. We considered that the other advantage of CMAs was to minimize the effect of MCC by skipping the culture process. Studies have demonstrated that array-CGH and SNParray can identify a mosaicism level of nearly $10 \%$ [38-41]. However, results from the current and other studies suggested that the low level of mosaicism was still difficult to detect with CMAs. We found an interesting case of multiple mosaic aneuploidy [arr(7)x3,(18)x3,(20)x3]. Conventional karyotyping enabled the identification of only one dominant trisomy. This rare complex aneuploidy may be associated with advanced maternal age and mitotic errors during the cleavage stage [42-45]. Given that the mother was 39 years old and had previously experienced four miscarriages, it is necessary to consider the etiology related to the postzygotic error as well as prezygotic error. Additional genetic counseling is needed to elucidate the cause of her repeated miscarriages based on the results of this study. Our study also has several limitations in that MCC was not fully discriminated from a normal female result because we did not compare it with maternal samples. Another limitation is that we could not perform FISH in all cases of mosaicism.

Taken together, no single technique can detect all genetic abnormalities, and conventional karyotyping should still be combined with other techniques to detect mosaicisms and balanced chromosomal rearrangements. FISH analysis can also be combined with other techniques to correctly discriminate true polyploidy. Nonetheless, SNP-array represents the first line of choice in clinic because the technique performs well in the detection of genetic abnormalities and minimizes the probability of testing failure as well as time, costs, and labor.

Supplementary Information The online version contains supplementary material available at https://doi.org/10.1007/s40291-021-00523-9.

\section{Declarations}

Funding The open access was funded by Dow Biomedica Inc., Korea. This research was supported by a grant (18172MFDS182) from the Ministry of Food and Drug Safety in 2021.
Conflict of interest J-ML, SYS, GWK, WJK, JHW, SBH, DK, HC, JY, YK, MK and IYP have no conflicts of interest that are directly relevant to the content of this article.

Availability of data and materials Not applicable.

Ethics approval The study was conducted in accordance with the ethical guidelines of the 1964 declaration of Helsinki and its later amendments or comparable ethical standards and was approved by the institutional review board/ethics committee of The Catholic University of Korea (KC18SESI0075).

Consent Written informed consent was obtained from all participants.

Author contributions Conception and design: J-ML, MK, and IYP. Patient data and samples: SYS, GWK, WJK, JHW, and SBH. Experiments, collection, and assembly of data: DK, HC, JY. Data analysis and interpretation: J-ML and MK. Manuscript writing and editing: J-ML, YK, MK, and IYP.

Open Access This article is licensed under a Creative Commons Attribution-NonCommercial 4.0 International License, which permits any non-commercial use, sharing, adaptation, distribution and reproduction in any medium or format, as long as you give appropriate credit to the original author(s) and the source, provide a link to the Creative Commons licence, and indicate if changes were made. The images or other third party material in this article are included in the article's Creative Commons licence, unless indicated otherwise in a credit line to the material. If material is not included in the article's Creative Commons licence and your intended use is not permitted by statutory regulation or exceeds the permitted use, you will need to obtain permission directly from the copyright holder. To view a copy of this licence, visit http://creativecommons.org/licenses/by-nc/4.0/.

\section{References}

1. Practice Committee of the American Society for Reproductive Medicine. Evaluation and treatment of recurrent pregnancy loss: a committee opinion. Fertil Steril. 2012;98(5):1103-11.

2. Sahoo T, Dzidic N, Strecker MN, Commander S, Travis MK, Doherty $\mathrm{C}$, et al. Comprehensive genetic analysis of pregnancy loss by chromosomal microarrays: outcomes, benefits, and challenges. Genet Med. 2017;19(1):83-9.

3. Menasha J, Levy B, Hirschhorn K, Kardon NB. Incidence and spectrum of chromosome abnormalities in spontaneous abortions: new insights from a 12-year study. Genet Med. 2005;7(4):251-63.

4. Kajii T, Ferrier A, Niikawa N, Takahara H, Ohama K, Avirachan S. Anatomic and chromosomal anomalies in 639 spontaneous abortuses. Hum Genet. 1980;55(1):87-98.

5. Dejmek J, Vojtaššák J, Malova J. Cytogenetic analysis of 1508 spontaneous abortions originating from south Slovakia. Eur J Obstetr Gynecol Reprod Biol. 1992;46(2-3):129-36.

6. Levy B, Sigurjonsson S, Pettersen B, Maisenbacher MK, Hall MP, Demko Z, et al. Genomic imbalance in products of conception: single-nucleotide polymorphism chromosomal microarray analysis. Obstetr Gynecol. 2014;124(2 PART 1):202-9.

7. Eiben B, Bartels I, Bähr-Porsch S, Borgmann S, Gatz G, Gellert $\mathrm{G}$, et al. Cytogenetic analysis of 750 spontaneous abortions with the direct-preparation method of chorionic villi and its implications for studying genetic causes of pregnancy wastage. Am J Hum Genet. 1990;47(4):656. 
8. Soler A, Morales C, Mademont-Soler I, Margarit E, Borrell A, Borobio V, et al. Overview of chromosome abnormalities in first trimester miscarriages: a series of 1,011 consecutive chorionic villi sample karyotypes. Cytogenet Genome Res. 2017;152(2):81-9.

9. Shah MS, Cinnioglu C, Maisenbacher M, Comstock I, Kort J, Lathi RB. Comparison of cytogenetics and molecular karyotyping for chromosome testing of miscarriage specimens. Fertil Steril. 2017;107(4):1028-33.

10. Lathi RB, Loring M, Massie JA, Demko ZP, Johnson D, Sigurjonsson S, et al. Informatics enhanced SNP microarray analysis of 30 miscarriage samples compared to routine cytogenetics. PLoS ONE. 2012;7(3):e31282.

11. Kaser D. The status of genetic screening in recurrent pregnancy loss. Obstetr Gynecol Clin. 2018;45(1):143-54.

12. Bell KA, Van Deerlin PG, Haddad BR, Feinberg RF. Cytogenetic diagnosis of "normal 46, XX" karyotypes in spontaneous abortions frequently may be misleading. Fertil Steril. 1999;71(2):334-41.

13. Battaglia A, Doccini V, Bernardini L, Novelli A, Loddo S, Capalbo A, et al. Confirmation of chromosomal microarray as a first-tier clinical diagnostic test for individuals with developmental delay, intellectual disability, autism spectrum disorders and dysmorphic features. Eur J Paediatr Neurol. 2013;17(6):589-99.

14. Miller DT, Adam MP, Aradhya S, Biesecker LG, Brothman AR, Carter NP, et al. Consensus statement: chromosomal microarray is a first-tier clinical diagnostic test for individuals with developmental disabilities or congenital anomalies. Am J Hum Genet. 2010;86(5):749-64.

15. Jang W, Kim Y, Han E, Park J, Chae H, Kwon A, et al. Chromosomal microarray analysis as a first-tier clinical diagnostic test in patients with developmental delay/intellectual disability, autism spectrum disorders, and multiple congenital anomalies: a prospective multicenter study in Korea. Ann Lab Med. 2019;39(3):299-310.

16. Reddy UM, Page GP, Saade GR. The role of DNA microarrays in the evaluation of fetal death. Prenat Diagn. 2012;32(4):371-5.

17. Strassberg M, Fruhman G, Van den Veyver IB. Copy-number changes in prenatal diagnosis. Expert Rev Mol Diagn. 2011;11(6):579-92.

18. Ballif BC, Kashork CD, Saleki R, Rorem E, Sundin K, Bejjani $\mathrm{BA}$, et al. Detecting sex chromosome anomalies and common triploidies in products of conception by array-based comparative genomic hybridization. Prenat Diagn. 2006;26(4):333-9.

19. Wang Y, Cheng Q, Meng L, Luo C, Hu H, Zhang J, et al. Clinical application of SNP array analysis in first-trimester pregnancy loss: a prospective study. Clin Genet. 2017;91(6):849-58.

20. Preisler J, Kopeika J, Ismail L, Vathanan V, Farren J, Abdallah Y, et al. Defining safe criteria to diagnose miscarriage: prospective observational multicentre study. BMJ (Clinical research ed). 2015;23(351):h4579.

21. McGowan-Jordan JSA, Schmid M, editors. ISCN: an international system for human cytogenomic nomenclature. Basel: Karger; 2016.

22. Jang W, Chae H, Kim J, Son J-O, Kim SC, Koo BK, et al. Identification of small marker chromosomes using microarray comparative genomic hybridization and multicolor fluorescent in situ hybridization. Mol Cytogenet. 2016;9(1):61.

23. Srebniak M, Boter M, Oudesluijs G, Joosten M, Govaerts L, Van Opstal D, et al. Application of SNP array for rapid prenatal diagnosis: implementation, genetic counselling and diagnostic flow. Eur J Hum Genet. 2011;19(12):1230-7.

24. South ST, Lee C, Lamb AN, Higgins AW, Kearney HM. ACMG Standards and Guidelines for constitutional cytogenomic microarray analysis, including postnatal and prenatal applications: revision 2013. Genet Med. 2013;15(11):901-9.
25. van den Berg MM, van Maarle MC, van Wely M, Goddijn M. Genetics of early miscarriage. Biochim Biophys Acta (BBA) Mol Basis Dis. 2012;1822(12):1951-9.

26. Hardy K, Hardy PJ. 1st trimester miscarriage: four decades of study. Transl Pediatr. 2015;4(2):189.

27. Geraedts J. Chromosomal anomalies and recurrent miscarriage. Infertil Reprod Med Clin N Am. 1996;7:677-88.

28. Warren JE, Silver RM. Genetics of pregnancy loss. Clin Obstet Gynecol. 2008;51(1):84-95.

29. Choi TY, Lee HM, Park WK, Jeong SY, Moon HS. Spontaneous abortion and recurrent miscarriage: a comparison of cytogenetic diagnosis in 250 cases. Obstetr Gynecol Sci. 2014;57(6):518.

30. Menasha J, Levy B, Hirschhorn K, Kardon NB. Incidence and spectrum of chromosome abnormalities in spontaneous abortions: new insights from a 12-year study. Genet Med. 2005;7(4):251-63.

31. Srinivas SK, Ma Y, Sammel MD, Chou D, McGrath C, Parry $\mathrm{S}$, et al. Placental inflammation and viral infection are implicated in second trimester pregnancy loss. Am J Obstet Gynecol. 2006;195(3):797-802.

32. Baud D, Regan L, Greub G. Emerging role of Chlamydia and Chlamydia-like organisms in adverse pregnancy outcomes. Curr Opin Infect Dis. 2008;21(1):70-6.

33. Allanson B, Jennings B, Jacques A, Charles AK, Dickinson JE. Infection and fetal loss in the mid-second trimester of pregnancy. Aust N Z J Obstet Gynaecol. 2010;50(3):221-5.

34. Rickman L, Fiegler H, Shaw-Smith C, Nash R, Cirigliano V, Voglino G, et al. Prenatal detection of unbalanced chromosomal rearrangements by array CGH. J Med Genet. 2006;43(4):353-61.

35. Fritz B, Hallermann C, Olert J, Fuchs B, Bruns M, Aslan M, et al. Cytogenetic analyses of culture failures by comparative genomic hybridisation ( $\mathrm{CGH}$ - - re-evaluation of chromosome aberration rates in early spontaneous abortions. Eur J Hum Genet. 2001;9(7):539-47.

36. Jarrett KL, Michaelis RC, Phelan MC, Vincent VA, Best RG. Microsatellite analysis reveals a high incidence of maternal cell contamination in $46, \mathrm{XX}$ products of conception consisting of villi or a combination of villi and membranous material. Am J Obstet Gynecol. 2001;185(1):198-203.

37. Lathi RB, Gustin SL, Keller J, Maisenbacher MK, Sigurjonsson $\mathrm{S}$, Tao R, et al. Reliability of 46 , XX results on miscarriage specimens: a review of 1,222 first-trimester miscarriage specimens. Fertil Steril. 2014;101(1):178-82.

38. Hoang S, Ahn J, Mann K, Bint S, Mansour S, Homfray T, et al. Detection of mosaicism for genome imbalance in a cohort of 3,042 clinical cases using an oligonucleotide array CGH platform. Eur J Med Genet. 2011;54(2):121-9.

39. Ballif BC, Rorem EA, Sundin K, Lincicum M, Gaskin S, Coppinger J, et al. Detection of low-level mosaicism by array $\mathrm{CGH}$ in routine diagnostic specimens. Am J Med Genet A. 2006;140(24):2757-67.

40. Biesecker LG, Spinner NB. A genomic view of mosaicism and human disease. Nat Rev Genet. 2013;14(5):307-20.

41. Conlin LK, Thiel BD, Bonnemann CG, Medne L, Ernst LM, Zackai EH, et al. Mechanisms of mosaicism, chimerism and uniparental disomy identified by single nucleotide polymorphism array analysis. Hum Mol Genet. 2010;19(7):1263-75.

42. Liu J, Wang W, Sun X, Liu L, Jin H, Li M, et al. DNA microarray reveals that high proportions of human blastocysts from women of advanced maternal age are aneuploid and mosaic. Biol Reproduct. 2012;87(6): 1-9 (148).

43. Rius M, Daina G, Obradors A, Ramos L, Velilla E, Fernández $S$, et al. Comprehensive embryo analysis of advanced maternal age-related aneuploidies and mosaicism by short comparative genomic hybridization. Fertil Steril. 2011;95(1):413-6. 
44. Fragouli E, Alfarawati S, Spath K, Jaroudi S, Sarasa J, Enciso M, et al. The origin and impact of embryonic aneuploidy. Hum Genet. 2013;132(9):1001-13.
45. Escudero T, Sumarroca M, Ribustello L, Munne S. Types of abnormalities in complex abnormal embryos. Fertil Steril. 2016;105(2):e19-20. 